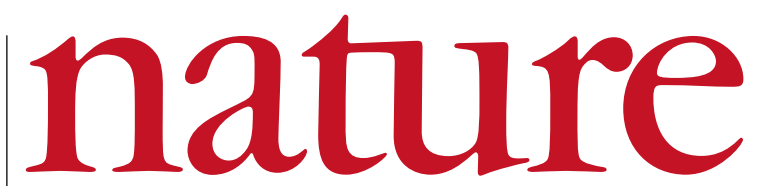

\title{
Poverty and transgenic crops
}

Africa's rejection of genetically modified food aid reflects a chasm of misunderstanding that is only exacerbated by exaggerated claims for the benefits of the technology.

A t first glance, the reluctance of some nations in southern Africa to accept international donations of genetically modified (GM) maize will strike some as bizarre and irresponsible. But the drama that is now being played out across the region (see page 571) raises some serious issues to which sceptics should pay heed, before they dismiss the problem as just another example of African governance gone awry.

The first issue is the extent to which aid donors like to enjoy most of the fruits of their own benevolence. In the case of US food aid, including some of the emergency aid currently flowing into southern Africa, grants or loans are normally made available only for the procurement of grain from US farmers. That makes the decision to grant the aid more politically palatable, because it is, in effect, just a few dollars more on top of the billions already being lavished on domestic farm support.

The second issue raised by the impasse is the extent to which transgenic crops are a relevant tool in eradicating poverty and feeding the world. Supporters of transgenic agriculture are engaged in an elaborate campaign to convince the public, particularly in Europe, that this is indeed the case. But the real impact of the technology on global poverty - now and for the foreseeable future - is to increase yields in rich countries, adding to a global grain glut that depresses prices and undermines agriculture in the poorest countries.

It is only in the longer term that transgenic technology holds out promise for these countries, and this will be fulfilled only if the countries concerned can introduce the technology on their own terms. The biosafety protocol of the Convention on Biological Diversity, for example, acknowledges the need for poor nations to develop the capacity to assess new agricultural technologies for themselves, if they are to use them effectively.

South Africa is the only country in sub-Saharan Africa that has been able to plan and implement rules for the commercial growing of GM food. Other countries in the region are now being pressed to accept the technology in an emergency, effectively without informed consent. Aid agencies cannot always prevent donated grain from being sold on the black market for planting by wealthier farmers in areas not afflicted by drought, they say, so the transgenic crops will arrive in their farms by default.

Countries in the region are understandably concerned about the trade implications of this. European consumers remain wary of GM food, and tighter labelling requirements for it are in the pipeline (see Nature 418, 114; 2002). Some countries are already targeting niche markets for non-GM food exports, and small African nations whose most lucrative potential export markets are in Europe - need to keep their options open.

It is certainly to be hoped that the United States is not using the current famine threat to get its GM crops into Africa through the back door to expand the restricted export market for them.

The United States donates almost $60 \%$ of the world's food aid and, as long as much of that aid is tied to the procurement of food from US farmers, the region facing famine will probably have to accept GM food. For now, negotiations are taking place to arrange the milling of GM maize before it arrives in Zimbabwe, to prevent the possibility of replanting. Some experts even suggest that the United States could exchange its GM maize with non-GM grain from a country prepared to accept both, such as India or South Africa, so that the latter can be distributed in the famished region. Such an arrangement might sound overelaborate, but it is only a taste of what is to come if Europe and the United States continue their mutual impasse on acceptance of this technology.

\section{Integrity from the top down}

\section{Research universities and other institutions are responsible for creating an environment that fosters scientific integrity.}

ast month, in a rare pronouncement by the US scientific establishment on a thorny subject, the Institute of Medicine (IOM) released a report on integrity in scientific research.

The IOM notes that fully fledged cases of scientific misconduct are rare. But it sensibly calls for research institutions to take a more active role in creating an environment where misconduct will not occur.

Every scientist would agree that good science requires solid experimental design, truthful and thorough reporting of results, honest peer review, good care of living subjects, and fairness to one's colleagues and students. The IOM report looks at what can be done to encourage researchers to adhere to such nostrums throughout their careers.

It concludes that government mandates are unlikely to imbue ethics. Nor are classes in which students are herded into a lecture hall to fulfil a requirement on a checklist. Advising and mentoring are crucial but, as the report states, the quality of mentors varies immensely. So it suggests that "both the call for change and its implementation must come from research institutions".
But having accepted that, what are universities to do? The IOM suggests that they teach research ethics creatively, as an integral part of core course content. It recommends that professional bodies evaluate institutional integrity as part of the accreditation process. However, no one knows how institutional characteristics influence research integrity, and the report calls for more research on this linkage.

This assessment comes hard on the heels of two recent cases of alleged misconduct, at the University of California, Berkeley, and at Bell Labs in Murray Hill, New Jersey. Institutions would be wise to take its premise seriously. Whenever a researcher commits major fraud, he or she has probably been getting away with smaller lapses for years - fudging a control here, deleting a messy data point there.

It is in the interests of every university and laboratory to help students think through the long-term consequences of what may at first appear to be minor violations of integrity. Ultimately, such early consideration will be good for institutions, good for the careers of young researchers, and good for science itself. 